\title{
VARIABILIDADE ESPACIAL DE PLANTAS DANINHAS NUM LATOSSOLO VERME- LHO SOB SISTEMA PLANTIO DIRETO'. \\ SPATIAL VARIABILITY OF WEEDS OF AN RED LATOSOL (OXISOL) UNDER NO- TILLAGE SYSTEM.
}

\author{
JOSILENE P. DE OLIVEIRA ${ }^{2}$, \\ LUCAS F. BATISTA ${ }^{3}$, \\ GLÉCIO M. SIQUEIRA4,*
}

\section{RESUMO:}

O objetivo deste trabalho foi analisar a variabilidade espacial de plantas daninhas num Latossolo Vermelho sob sistema plantio direto. As medições foram realizadas em uma área de $0,5 \mathrm{ha}$, em 50 pontos de amostragem com espaçamento de $5 \mathrm{~m} \times 10 \mathrm{~m}$. Os dados foram analisados por meio da estatística clássica e da geoestatística, e os mapas de variabilidade espacial construídos por meio da técnica de interpolação por krigagem. Todas as espécies de plantas daninhas presentes na área de estudo apresentaram variabilidade espacial, com exceção da corda-de-viola (Ipomoea triloba L.) e crista-de-galo (Heliotropium indicum L.), que apresentaram efeito pepita puro. Os valores de alcance (a) demonstram que o espaçamento entre amostras pode ser ampliado para todas as espécies de plantas daninhas. Não foi possível determinar zonas específicas de manejo na área de estudo uma vez que as distintas espécies de plantas daninhas infestaram diferentes zonas da área de estudo.

PALAVRAS-CHAVES: Manejo Localizado; Semivariogramas; Agricultura de Precisão.

\footnotetext{
${ }^{1}$ Projeto de Iniciação Científica do primeiro e segundo autor realizado em Cooperação entre o Instituto Federal GoianoCampus de Urutaí (Goiás, Brasil) e a Universidade de Santiago de Compostela (Galícia, Espanha).

${ }^{2}$ Graduando em Tecnologia em Irrigação e Drenagem, Instituto Federal Goiano Campus de Urutaí, Rodovia Geraldo S. Nascimento, km 2,5, Urutaí, Goiás, Brasil. josi_op@hotmail.com.

${ }^{3}$ Graduando em Medicina Veterinária, Universidade Federal de Goiás, Campus Samambaia, 74001-970, Goiânia, Goiás, Brasil. lucaslfb@hotmail.com.

${ }^{4}$ Universidade de Santiago de Compostela, Escuela Politécnica Superior s/n, 27002, Lugo, España. * Autor correspon dente.glecio.machado@rai.usc.es
} 


\section{ABSTRACT:}

The objective of this study was to analyze the spatial variability of weeds in an Red Latosol (Oxisol) under no-tillage system. Samples were taken in an area of 0.5 ha, in 50 sampling points with spacing of $5 \mathrm{~m} \times 10 \mathrm{~m}$. Data were analyzed by means of classical statistics and geostatistics, and spatial variability of the maps constructed by the technique of interpolation by kriging. All species of weeds present in the study area showed spatial variability with the exception of Ipomoea triloba (L.) and Heliotropium indicum (L.), which showed pure nugget effect. The values of range (a) show that the spacing between samples can be extended to all species of weeds. Unable to determine specifics areas of management in the local since the different species of weed infested areas of different area.

KEYWORDS: Site Specific Management; Semivariograms, Precision Agriculture.

\section{INTRODUÇÃO}

O conhecimento de como as populações de plantas daninhas se desenvolvem permite agregar ao sistema de produção agrícola uma série de informações que até então eram ignoradas, uma vez que geralmente a aplicação dos herbicidas é realizada considerando um valor médio de infestação para toda a área de cultivo. Assim, o monitoramento espacial e temporal da variabilidade das plantas daninhas permite a prática da agricultura de precisão, por meio do mapeamento das zonas de infestação e da aplicação localizada dos herbicidas.

O estudo da variabilidade espacial das zonas de infestação das plantas daninhas permite a determinação de zonas específicas de manejo. De acordo com MORTENSEN et al. (1998) as espécies de plantas daninhas apresentam estabilidade temporal, o que favorece o processo de gestão das áreas de cultivos. No entanto, no Brasil pouco se conhece sobre a variabilidade espacial das plantas daninhas. Nesse contexto, podemos citar os trabalhos de SHIRATSUCHI et al. (2004, 2005), SCHAFFRATH et al. (2007) e MONQUERO et al. (2008) que estudaram a distribuição espacial de plantas daninhas com o objetivo de determinar zonas de manejo específico. SHIRATSUCHI et al. (2005) descrevem ainda que a maioria dos trabalhos que enfocam o mapeamento de plantas daninhas diz respeito ao mapeamento da flora emergente durante o ciclo crítico de prevenção da interferência, havendo assim escassos trabalhos sobre a varia- bilidade espacial das plantas daninhas no decorrer do ciclo dos cultivos.

Desta maneira, este trabalho teve como objetivo determinar a variabilidade espacial de plantas daninhas num Latossolo Vermelho manejado sob o sistema plantio direto em Urutaí (Goiás) numa área de Cerrado.

\section{MATERIAL E MÉTODOS}

A área de estudo possui 0,5 ha $(50 \mathrm{~m} \mathrm{x}$ $100 \mathrm{~m}$ ) e está localizada no Instituto Federal Goiano - Campus de Urutaí ( $17^{\circ} 27^{\prime} 50^{\prime \prime}$ Sul e $48^{\circ} 12^{\prime} 10^{\prime \prime}$ Oeste). O solo da área é um Latossolo Vermelho eutroférrico, textura argilosa (EMBRAPA, 2006) manejado sob plantio direto desde 2001 e no momento das amostragens estava cultivado com girassol (Helianthus annuus L.) cultivar M-734 semeado no dia 06/03/2009 com espaçamento de 0,25 $\mathrm{m} \times 0,90 \mathrm{~m}$. O clima, de acordo com a classificação de Köppen é Aw, com duas estações bem definidas, seca no inverno e úmida no verão, com temperatura média durante todos os meses do ano superior a $18^{\circ} \mathrm{C}$.

No dia 27/03/2009 a área de estudo foi dividida em uma grade de amostragem contendo 50 pontos com espaçamento de $5 \mathrm{~m} \times 10 \mathrm{~m}$ (Figura 1). Em cada ponto de amostragem foi lançado ao acaso um circulo com $0,5 \mathrm{~m}$ de diâmetro $(0,196 \mathrm{~m} 2)$ para identificação do número de indivíduos por ponto, número de espécies 
por ponto e a incidência de cada espécie em cada um dos pontos de amostragem, por meio da técnica de identificação manual (LUTMAN \& PERRY, 1999). A identificação das plantas daninhas presentes na área de estudo foi realizada utilizando o Manual de Identificação e Controle de Plantas Daninhas (LORENZI, 2000), sendo identificadas as seguintes espécies de plantas daninhas: carrapicho (Cenchurus echinatus L.), burra leiteira [Chamaesyce sp. (L.) Mill], crista-de-galo (Heliotropium indicum L.), corda-de-viola (Ipomoea triloba L.), capim-péde-galinha [Eleusine indica (L.) Gaertn] e picãopreto (Bidens pilosa L.).

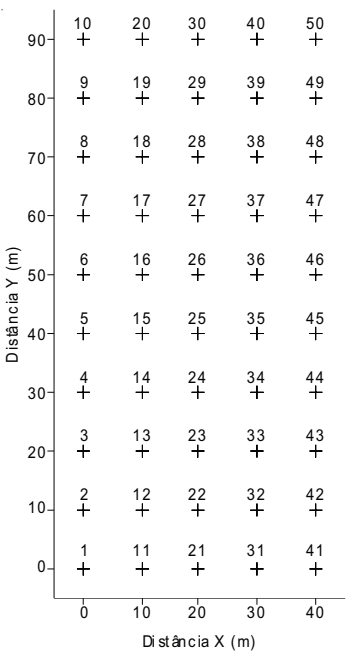

Figura 1. Esquema de coleta de amostras na área de estudo, contendo 50 pontos com espaçamento regular de $5 \mathrm{~m} \times 10 \mathrm{~m}$.

A variabilidade espacial foi analisada por meio da construção de semivariogramas, de acordo com VIEIRA (2000).O semivariograma, $y(h)$, de uma variável distribuída espacialmente, $y(h)$,é calculado conforme a Equação 1:

$$
\gamma(h)=\frac{1}{2 N(h)} \sum_{i=1}^{N(h)}\left[z\left(x_{i}\right)-z\left(x_{i}+h\right)\right]^{2}
$$

onde $N(h)$ é o número de observações separadas por uma distância $h$. Os semivariogramas foram ajustados a um modelo matemático de acordo com os seguintes parâmetros: efeito pepita (C0), patamar $(\mathrm{C} 0+\mathrm{C} 1)$, e alcance (a). A Equação 1 foi determinada considerando a hipótese intrínseca da geoestatística, dentro da qual não há nenhuma exigência para a existên- cia de uma variância finita, $\operatorname{Var}(z)$. Exige-se apenas a estacionaridade da média e a estacionaridade de segunda ordem das diferenças $[(z(x)-z(x+h)]$ (JOURNEL \& HUIJBREGTS, 1978). O comportamento do semivariograma para valores pequenos de $\mathrm{h}$ revela aspectos muito importantes da variabilidade espacial das propriedades em estudo os quais podem ser utilizados para comparação.

Os semivariogramas das plantas daninhas foram escalonados, de acordo com VIEIRA et al. (1997):

$$
\gamma^{s c}(h)=\frac{\gamma(h)}{\operatorname{Var}(z)}
$$

onde $y^{s c}(h)$ é o semivariograma escalonado, $y(h)$ é o semivariograma original, e $\operatorname{Var}(z)$ é a variância dos dados. Teoricamente, esta equação requer a existência de uma variância finita, a qual pode ser garantida se a estacionaridade de segunda ordem existir. Entretanto, a grandeza que se utiliza neste cálculo é apenas o número convenientemente calculado para variância dos dados, mas não exatamente a grandeza estatística variância. O escalonamento é utilizado com a finalidade de desenhar vários semivariogramas no mesmo gráfico, quando de outra maneira teriam escalas diferentes no eixo das semivariancias. Quando semivariogramas escalonados se agrupam pode-se dizer que as propriedades envolvidas tem variabilidade espacial semelhante (VIEIRA et al., 1997). O ajuste do semivarograma experimental das plantas daninhas foi realizado ajustando os modelos esférico, exponencial e gaussiano, sendo o melhor ajuste escolhido em função da técnica de "jack-knifing", conforme apresentado por CARVALHO et al. (2002).

A razão de dependência espacial entre amostras foi determinada conforme proposto por CAMBARDELLA et al. (1994, Equação 3), sendo $0,00-0,25 \%$ forte, de 25,00-75,00 moderada e de $75,00-100,00 \%$ fraca.

$$
R D=\left(\frac{C_{0}}{C_{0}+C_{1}}\right) \times 100
$$


A técnica de interpolação por krigagem foi utilizada antes da construção de mapas, a fim de providenciar uma quantidade suficiente de informações e, assim, evitar estimativas de má qualidade e confiança. A construção dos mapas de isolinhas da variabilidade espacial para os atributos analisados foi feita usando o software SURFER 7.0 (GOLDEN SOFTWARE, 1999), onde os valores foram determinados para os locais não amostrados pela técnica de krigagem segundo VIEIRA (2000).

\section{RESULTADOS E DISCUSSÃO}

A maioria das espécies de plantas daninhas estudadas apresentaram distribuição de freqüência do tipo lognormal, com exceção do número de espécies que apresentou normalidade dos dados por meio do teste de Kolmogorov-Smirnov com probabilidade de erro de $1 \%$ (D, Tabela 1). De acordo com CARVALHO et al. (2002), valores de assimetria e curtose próximos a 0 e 3 são um indicativo de distribuição de freqüência normal. Os elevados valores de assimetria e curtose confirmam a presença de distribuição lognormal para a maioria das espécies de plantas daninhas.

De acordo com a classificação de WARRICK \& NIELSEN (1980) o número de indivíduos, o carrapicho, a crista-de-galo e o capim pé-de-galinha apresentam elevados valores de coeficiente de variação ( $C V \geq 60,00 \%$ ), as demais variáveis apresentam moderados valores de $\mathrm{CV}$. O carrapicho foi à espécie de planta daninha mais comum na área de estudo, ocorrendo em 41 pontos dos 50 pontos de amostragem, e a corda-de-viola a espécie menos freqüente na área de estudo sendo encontrada em apenas 5 pontos de amostragem.

Tabela 1. Parâmetros estatísticos das plantas daninhas presentes na área de estudo.

\begin{tabular}{ccccccccc}
\hline Atributos & $\mathbf{N}$ & Mín. & Máx. & Média & CV & Assim. & Curt. & D \\
\hline Número de indivíduos & 48 & 2,00 & 40,00 & 11,81 & 69,34 & 1,16 & 1,53 & $0,231 \mathrm{Ln}$ \\
Número de espécies & 48 & 1,00 & 6,00 & 2,43 & 40,50 & 0,91 & 1,97 & $0,231 \mathrm{n}$ \\
$\quad$ Carrapicho & 41 & 1,00 & 40,00 & 8,90 & 85,06 & 2,01 & 6,36 & $0,249 \mathrm{Ln}$ \\
Burra leiteira & 10 & 1,00 & 3,00 & 1,50 & 56,65 & 1,36 & 0,11 & $0,489 \mathrm{Ln}$ \\
Crista-de-galo & 13 & 1,00 & 7,00 & 2,50 & 80,59 & 1,20 & 0,60 & $0,432 \mathrm{Ln}$ \\
Corda-de-viola & 5 & 1,00 & 2,00 & 1,20 & 37,26 & 2,24 & 5,00 & $0,669 \mathrm{Ln}$ \\
Pé-de-galinha & 20 & 1,00 & 10,00 & 4,00 & 81,88 & 0,87 & $-0,84$ & $0,352 \mathrm{Ln}$ \\
Picão preto & 25 & 1,00 & 4,00 & 2,60 & 46,60 & $-0,15$ & $-1,51$ & $0,317 \mathrm{Ln}$ \\
\hline
\end{tabular}

$\mathrm{N}$ : número de medidas; Mín.: valor mínimo; Máx.: valor máximo; CV: Coeficiente de variação; Assim.: assimetria; Curt.: curtose; D: desvio máximo em relação a média por meio do teste de Kolmogorov-Smirnov com probabilidade de erro de $1 \%$. 
A matriz de correlação linear (Tabela 2) demonstra que entre Número de indivíduos $\mathrm{x}$ Carrapicho $(r=0,872)$, Número de indivíduos $\mathrm{X}$ Crista-de-galo $(r=0,730)$, Número de indivíduos x Corda-de-viola $(r=50,549)$, Número de espécies $\times$ Crista-de-galo $(r=0,770)$, Carrapicho $\mathrm{x}$ Corda-de-viola $(r=-0,783)$, Burra leiteira $\mathrm{x}$
Picão preto $(r=0,520)$ e Crista-de-galo x Péde-galinha $(r=0,872)$ existe uma elevada correlação linear conforme a classificação de SANTOS (2007). As demais correlações são consideradas baixas $(|r|=0,1-0,5)$ ou nulas $(|r|=<$ $0,1)$.

Tabela 2. Correlação linear para entre as espécies de plantas daninhas presentes na área de estudo.

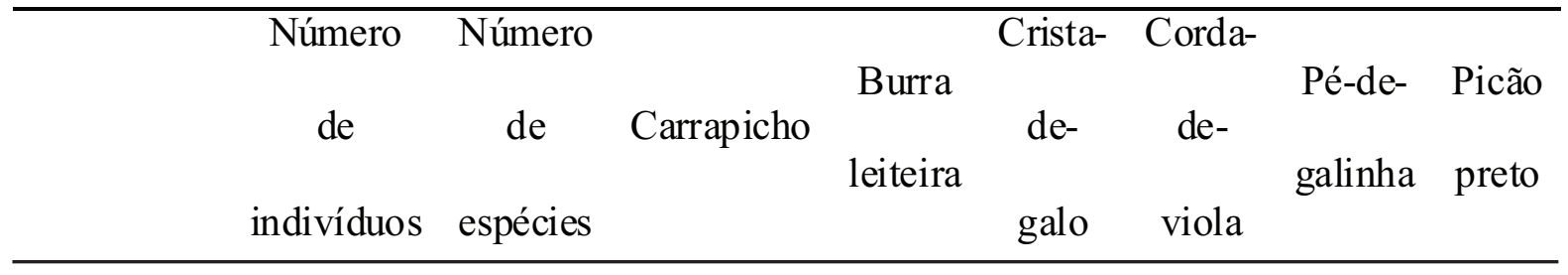

Número

de $\quad 1,000$

indivíduos

Número

de $\quad 0,462 \quad 1,000$

espécies

Carrapicho $\quad 0,872 \quad 0,054 \quad 1,000$

Burra

leiteira

$$
0,162 \quad 0,243 \quad-0,162 \quad 1,000
$$

Crista-de-

galo

$$
0,730 \quad 0,770 \quad 0,123 \quad 0,000 \quad 1,000
$$

Corda-de-

$$
\begin{array}{llllll}
-0,549 & 0,085 & -0,783 & 0,000 & 0,000 & 1,000
\end{array}
$$

viola

Pé-de-

galinha

$$
\begin{array}{lllllll}
0,353 & 0,092 & -0,170 & 0,480 & 0,820 & 0,000 & 1,000
\end{array}
$$

Picão

$$
\begin{array}{llllllll}
-0,241 & -0,298 & -0,371 & 0,520 & 0,190 & 0,000 & -0,040 & 1,000
\end{array}
$$

preto 
A presença de correlação linear negativa para a grande maioria das espécies com o capim carrapicho (Carrapicho $\times$ Corda-de-viola $=$ $-0,783$; carrapicho $\times$ Burra leiteira $=-0,162$; Carrapicho x Pé-de-galinha = -0,170; Carrapicho $x$ Picão preto $=-0,781$ ) indica a superioridade do capim carrapicho no processo de colonização da área de estudo em relação as demais espécies de plantas daninhas, este fato é confirmado quando analisamos a ocorrência de cada uma das espécies de plantas daninhas nos 50 pontos de amostragem (Tabela 1).

A análise geoestatística demonstrou que o número de espécies, a crista-de-galo e a corda-de-viola apresentaram efeito pepita puro (Tabela 3). De acordo com VIEIRA (2000) e SIQUEIRA et al. (2008) a presença de efeito pepita se deve principalmente porque o espaçamento utilizado não foi suficiente para detectar a variabilidade espacial entre amostras. No entanto, a presença de efeito pepita puro para a crista-de-galo e a corda-de-viola se deve principalmente porque estas duas espécies de plantas daninhas não são freqüentes na área de estudo, conforme demonstra o número de medidas (Tabela 1).

O número de indivíduos e o carrapicho apresentaram elevados valores de efeito pepi- ta (C0, Tabela 3). SIQUEIRA et al. (2008) descrevem que os valores de efeito pepita representam a variabilidade espacial não detectada no processo de amostragem, indicando que se o espaçamento fosse menor seria possível detectar outros padrões de variabilidade espacial para estes atributos.

O modelo esférico foi o que mais se ajustou aos dados de plantas de daninhas, corroborando com outros estudos que descrevem este modelo como o que mais se ajusta aos dados de solo e planta (CAMBARDELLA et al., 1994; VIEIRA, 2000; SIQUEIRA et al., 2008), com exceção do capim carrapicho que se ajustou ao modelo gaussiano.

O capim pé-de-galinha apresentou o maior valor de alcance $(a=40,00 \mathrm{~m})$ e o número de indivíduos e o picão preto apresentaram o menor valor de alcance $(a=20,00 \mathrm{~m})$. A razão de dependência espacial calculada conforme CAMBARDELLA et al. (1994) é elevada (RD ? $25,00 \%$ ) para a burra leiteira ( $R D=0,00 \%)$, o capim pé-de-galinha ( $R D=0,00 \%)$ e o picão preto $(\mathrm{RD}=7,14 \%$ ). O número de indivíduos e o capim carrapicho apresentaram moderada razão de dependência espacial $(R D=25,00$ $75,00 \%$ ).

Tabela 3. Parâmetros de ajuste do semivariograma para as espécies de plantas daninhas estudadas.

\begin{tabular}{|c|c|c|c|c|c|}
\hline & Modelo & $\mathrm{C}_{0}$ & $\mathrm{C}_{1}$ & $\bar{a}$ & $\mathrm{RD}$ \\
\hline Número de indivíduos & Esférico & 40,00 & 28,00 & 20,00 & 58,82 \\
\hline Número de espécies & \multicolumn{5}{|c|}{ Efeito pepita puro } \\
\hline Carrapicho & Gaussiano & 25,00 & 60,00 & 38,00 & 29,41 \\
\hline Burra leiteira & Esférico & 0,00 & 0,60 & 28,00 & 0,00 \\
\hline Crista-de-galo & \multicolumn{5}{|c|}{ Efeito pepita puro } \\
\hline Corda-de-viola & \multicolumn{5}{|c|}{ Efeito pepita puro } \\
\hline Pé-de-galinha & Esférico & 0,00 & 15,00 & 40,00 & 0,00 \\
\hline Picão preto & Esférico & 0,10 & 1,30 & 20,00 & 7,14 \\
\hline
\end{tabular}

C0: efeito pepita; C1: variância estrutural; a: alcance (m); RD: razão de dependência espacial. 
A Figura 2 apresenta o semivariograma experimental para cada uma das variáveis em estudo. Percebe-se que existe um padrão de variabilidade espacial similar para as variáveis que se ajustaram ao modelo matemático esférico, com exceção do capim pé-de-galinha que apresenta uma menor variabilidade entre os pares de semivariância principalmente na pequena distância. No entanto, a análise do semivariograma escalonado (Figura 3) demonstra que de maneira geral todas as variáveis que apresentaram variabilidade espacial têm o mesmo comportamento espacial, exceto a burra leiteira, a crista-de-galo e a corda-de-viola que apresentam grande dispersão dos pares de semivariância na pequena distância e com o aumento da distância.
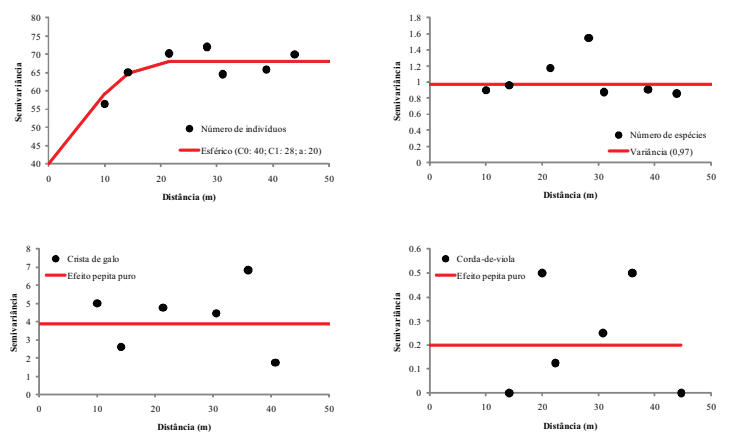
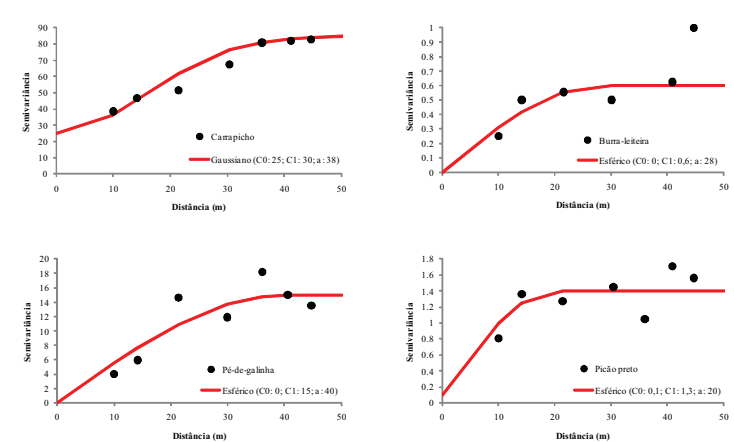

Figura 2. Semivariogramas ajustados para as plantas daninhas presentes na área de estudo.

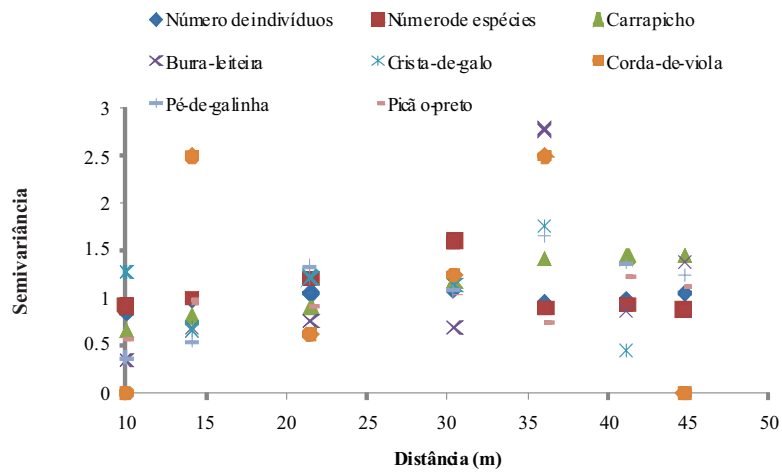

Figura 3. Semivariograma escalonado para as espécies de plantas daninhas estudadas.

Os mapas de variabilidade espacial (Figura 4) demonstram que existe uma grande semelhança na distribuição das isolinhas do mapa do número de indivíduos e do capim carrapicho, este fato já era esperado uma vez que o capim carrapicho é espécie de planta daninha que mais ocorre na área de estudo, sendo encontrada em 41 pontos (Tabela 1) dos 50 pontos de amostragem.

O mapa de variabilidade espacial do capim carrapicho demonstra que esta espécie de planta daninha esta presente em toda a área de estudo, ocorrendo com maior intensidade na parte lateral esquerda da área de estudo.
O capim pé-de-galinha e o picão preto apresentam distintos padrões de incidência, todavia se pode notar que estas espécies de plantas daninhas estão presentes principalmente na parte lateral direita da área de estudo. A burra leiteira apresenta uma maior incidência na parte inferior da área (Figura 4). 
Os mapas de variabilidade espacial do capim pé-de-galinha e do picão preto demonstram zonas de incidência comuns na parte superior da área de estudo. De maneira geral, os mapas de variabilidade espacial demonstram que não existe uma coincidência no padrão de distribuição espacial das espécies de plantas daninhas (carrapicho, burra leiteira, pé-de-galinha e picão preto) na área de estudo, o que dificulta o delineamento de zonas de manejo diferenciado.
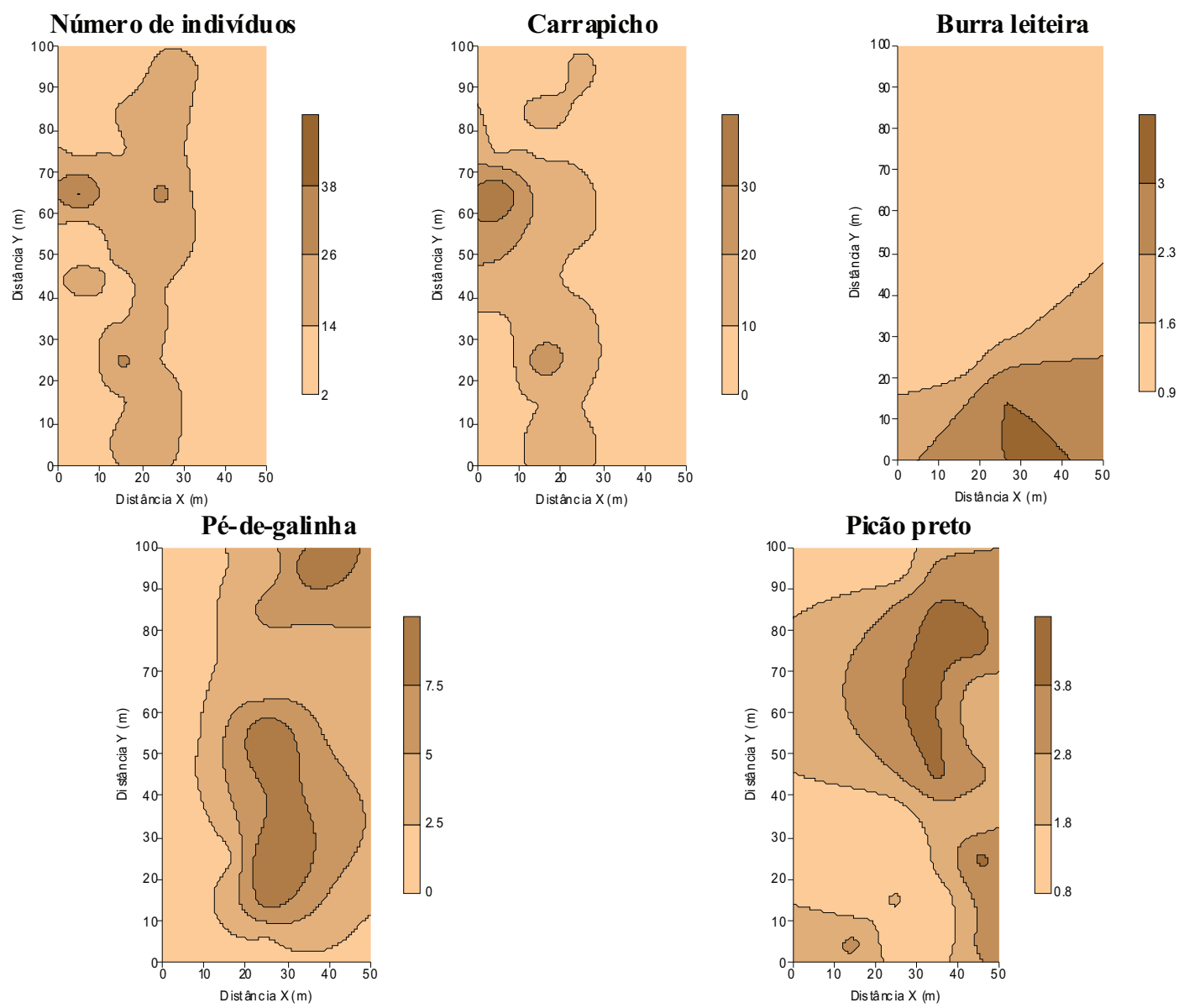

Figura 4. Mapas de variabilidade espacial para as espécies de plantas daninhas presentes na área de estudo.

\section{CONCLUSÕES}

Todas as espécies de plantas daninhas presentes na área de estudo apresentaram variabilidade espacial com exceção da corda-de-viola e crista-de-galo. As espécies de plantas daninhas apresentaram distribuição em reboleiras. Os valores de alcance (a) demonstram que o espaçamento entre amostras pode ser ampliado para todas as espécies de plantas daninhas. Não foi possível determinar zonas específicas de manejo na área de estudo uma vez que as distintas espécies de plantas daninhas infestaram diferentes zonas da área de estudo.

\section{BIBLIOGRAFIA}

CAMBARDELLA, C.E.; MOORMAN, T.B.; NOVAK, J.M.; PARKIN, T.B.; KARLEN, D.L.; TURCO, R.F.; KONOPKA, A.E. Field-scale variability of soil properties in Central lowa soils. Soil Science Society of America Journal, 58:1501-1511, 1994.

CARVALHO, J.R.P.; SILVEIRA, P.M. \& VIEIRA, S.R. Geoestatística na determinação da variabilidade espacial de características químicas do solo sob diferentes preparos. Pesquisa Agropecuária Brasileira, v.37, n.8, p.1151-1159, 2002. 
EMBRAPA. Centro Nacional de Pesquisa de Solos, Sistema Brasileiro de Classificação de Solos, 2,ed, Rio de Janeiro, 2006. 306pp, GOLDEN SOFTWARE. Surfer: User's Guide (Versión 7.0). EEUU: Golden Software, 1999. $679 \mathrm{p}$.

JOURNEL, A.G.; HUIJBREGTS, Ch.J. Mining geostatistics. London, Academic Press, 1978. $600 p$.

LORENZI, H. Manual de identificação e controle de plantas daninhas: plantio direto e convencional. 5.ed, Nova Odessa: Plantarum, 2000. $384 \mathrm{p}$.

LUTMAN, P.J.W.; PERRY, N.H. Methods of weed patch detection in cereal crops, In: The 1999 Brighton Conference - Weeds, Brighton 1999, Proceedings, Brighton: BCPC, 1999, p.627-634.

MONQUERO, P.A.; AMARAL, L.R.; BINHA, D.P.; SILVA, P.V.; SILVA, A.C.; MARTINS, F.R.A. Mapas de infestação de plantas daninhas em diferentes sistemas de colheita da cana-de-açúcar. Planta Daninha, Viçosa, v.26, n.1, p. 47-55, 2008.

MORTENSEN, D.A.; DIELEMANN, J.A.; JOHNSON, G.A. Weed spatial variation and weed management. In: HATFIELD, J.L., BUHLERARD, D.D., STEWART, B.A. (Eds.), Integrated Weed and Soil Management. Ann Arbor Press, Chelsea, MI, 1998. pp. 293-309. SANTOS, C.M.A. Estatística Descritiva - Manual de Auto-aprendizagem, Lisboa, Edições Sílabo, 2007. 261p.

SCHAFFRATH, V.R.; TORMENA, C.A.; GONÇALVES, A.C.A.; OLIVEIRA JUNIOR, R.S. Variabilidade espacial de plantas daninhas em dois sistemas de manejo de solo. Revista Brasileira de Engenharia Agrícola e Ambiental, Campina Grande, v.11, n.1, p.53-60, 2007.

SHIRATSUCHI, L.S.; FONTES, J.R.A.; RESENDE, A.V. Correlação da distribuição espacial do banco de sementes de plantas daninhas com a fertilidade dos solos. Planta Daninha, Viçosa, v. 23, n. 3, p. 429-436, 2005.
SHIRATSUCHI, L.S.; MOLIN, J.P.; CHRISTOFFOLETI, P.J. Mapeamento da distribuição espacial da infestação de Panicum maximum durante a colheita da cultura de miIho. Planta Daninha, Viçosa, v.22, n.2, p.269274, 2004.

SIQUEIRA. G.M.; VIEIRA, S.R.; CEDDIA, M.B. Variabilidade espacial de atributos físicos do solo determinados por métodos diversos. Bragantia, Campinas, v.67, n.1, p.203-211, 2008.

VIEIRA, S.R. Geoestatística em estudos de variabilidade espacial do solo. In: NOVAIS, R.F., ALVAREZ, V.H., SCHAEFER, G.R. (ed.) Tópicos em Ciência do solo. Viçosa: Sociedade Brasileira de Ciência do Solo, v.1, 2000. p. 1-54.

VIEIRA, S.R.; NIELSEN, D.R.; BIGGAR, J.W.; TILLOTSON, P.M. The Scaling of semivariograms and the kriging estimation. Revista Brasileira de Ciência do Solo, v.21, p.525-533, 1997.

WARRICK, A.W.; NIELSEN, D.R. Spatial variability of soil physical properties in the field. In: HILLEL, D. Applications of soil physics, New York: Academic Press, 1980. 